\title{
AN INFRARED EXPERIMENTAL APPROACH TO VISUALIZE THERMAL IRREGULARITIES IN HISTORICAL BUILDING MANSONRY WALLS
}

\author{
F. Fantozzi, S. Filippeschi, F. Leccese \\ Department of Energetics "Lorenzo Poggi" - University of Pisa \\ Via Diotisalvi 2, 56100 Pisa, Italy \\ tel +39-050-2217153 fax +39-050-2217150 \\ s filippeschi@ing unipi.it;g salva@ing unipi it
}

The infrared thermography represents a very useful non-destructive technique in the building diagnostics. In particular, the technical literature reports several "qualitative" criteria for the detection of thermal irregularities in building envelope are reported with regard to thermal insulation defects, air leakages and moisture effects.

In the last few years this technique has been widely used in the cultural heritage preservation field both with the solar heating (passive) and with heating devices (active). The flexibility of the passive infrared thermal method can depend, even significantly, on the environmental conditions (i.e. exposure of the walls of the building, climatic conditions, walls colours and decay).

On the other hand the most of the historical buildings under restoration are generally not occupied, not heated and in hard-decay situations, concerning both exterior envelope and interior partitions. In these cases a simple qualitative analysis can provide bad results and the operator's experience turns out to be crucial during the shooting procedure in situ.

All the quantitative analysis are based on the measurement of the temperature of sample over time and their successive images processing. The most of thermal quantitative methods reported in literature has been developed for industrial applications. In case of historical building the thermal properties of the materials are very different and the time of heating and thermal infrared observation is longer.

Moreover the materials found in historical walls are very different among them and, therefore, thay are different also by a reference case previously analysed in laboratory or reported in literature.

In literature a significant database containing experimental data deduced from images in situ is still lacking owing both to the recent application of these techniques to the specific field and to the difficulty in processing images according to standard procedures and in cleaning up the thermal signal by eliminating any measurement error (noises).

In this paper the authors show several experiences on infrared thermal tests of historical buildings in Tuscany realized by using a LW infrared camera with microbolometric sensor. The obtained thermographic images have been processed using the thermal contrast as referent parameter. The images have been corrected in order to taking in account the different angles of view.

In order to define the minimum thermal contrast that allows a good definition in the analysis of the texture. The images have been analyzed by proposing a quantitative analysis of active techniques (Step heating with oil burner) based on the comparison of the temperature evolutions of a wall experimentally measured and numerically simulated with simple algorithms.

The mathematical model is based on the idea that a portion of mortar and a portion of a stone, as a thermal radiation is supplied, show thermal evolutions that are not influenced by boundary and, therefore, a lumped capacitance method can be used. In particular have been neglected also the thermal diffusion in the plaster thickness. 
The mathematical model has been developed in case of active heating, that in the experimental cases in situ has been realised by an oil burner heater. The most of heat supplied by the oil burn heat to the wall is in radiating mode, so that the natural convection can be neglected in the first hypothesis.

The mathematical model that have been compared with the experimental evolutions are three. The first method takes in account a wall made of a portion of mortar and a portion of another material (stone, whole brick, hollow brick, etc) both approximated as semi-infinitive medium. The second configuration add to the previous wall a thick of plaster which is approximated with a finite difference method both in space and in time.

This simple method has been compared with a wall made a layer of plaster and a layer of mortar and another material but this wall has been approximated with a finite difference method both implicit and explicit. These results have been compared with the experimental one. The mathematical model calculates the thermal contrast between the mortar and the other material which varies over time on the external layer of the plaster.

In figure 1 is reported the thermal evolutions of the thermal contrast for a wall made of whole bricks and the reference mortar. All the numerical results have been compared with in situ evolutions of walls. The paper shows the numerical results of this calculations made with different material (different kind of stones, hollow and whole bricks, woods, etc.). Interesting considerations can be drawn on the time in which the different materials which made the wall "appear" on the external face of the wall. The "appearing time" is time for reaching a sufficient thermal contrast on the external face of the wall. The sufficient thermal contrast depends on the thermal resolution of the thermograhpic instruments.

The preliminary results on a sensibility analysis of the different parameters being able to influence the "appearing time" of different materials has been shown in this paper.

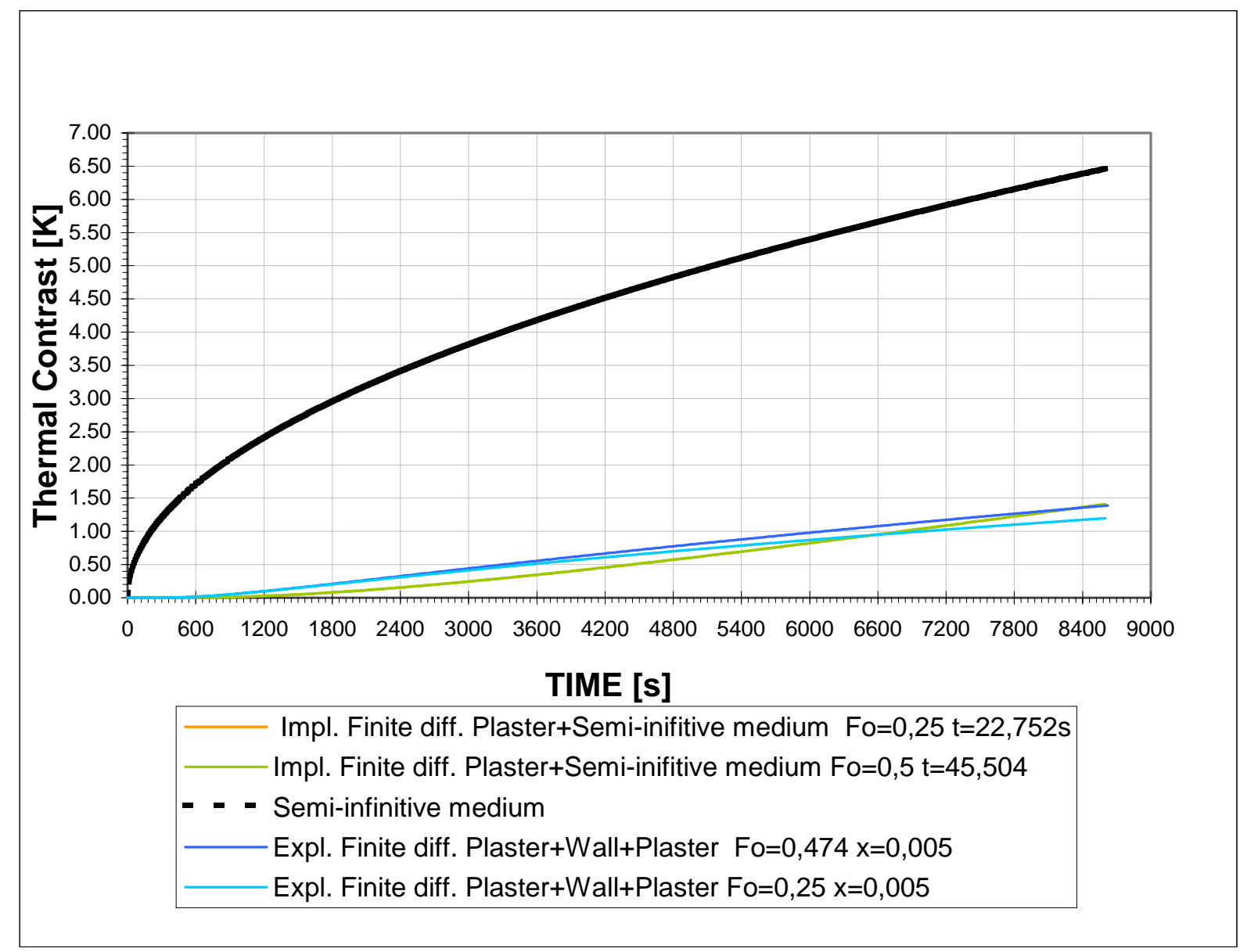

